\title{
An Alternative Method to Compute the Bit Error Probability of Modulation Schemes Subject to Nakagami- $m$ Fading
}

\author{
Wamberto J. L. Queiroz, ${ }^{1}$ Waslon T. A. Lopes, ${ }^{1}$ Francisco Madeiro, ${ }^{2}$ and Marcelo S. Alencar ${ }^{1}$ \\ ${ }^{1}$ Departamento de Engenharia Elétrica, Universidade Federal de Campina Grande, 58.429-900, Campina Grande, PB, Brazil \\ ${ }^{2}$ Escola Politécnica de Pernambuco, Universidade de Pernambuco, 50.750-470, Recife, PE, Brazil
}

Correspondence should be addressed to Marcelo S. Alencar, malencar@iecom.org.br

Received 4 March 2010; Revised 23 June 2010; Accepted 24 September 2010

Academic Editor: Athanasios Rontogiannis

Copyright ( 2010 Wamberto J. L. Queiroz et al. This is an open access article distributed under the Creative Commons Attribution License, which permits unrestricted use, distribution, and reproduction in any medium, provided the original work is properly cited.

\begin{abstract}
This paper presents an alternative method for determining exact expressions for the bit error probability (BEP) of modulation schemes subject to Nakagami- $m$ fading. In this method, the Nakagami- $m$ fading channel is seen as an additive noise channel whose noise is modeled as the ratio between Gaussian and Nakagami- $m$ random variables. The method consists of using the cumulative density function of the resulting noise to obtain closed-form expressions for the BEP of modulation schemes subject to Nakagami$m$ fading. In particular, the proposed method is used to obtain closed-form expressions for the BEP of $M$-ary quadrature amplitude modulation ( $M$-QAM), $M$-ary pulse amplitude modulation $(M$-PAM), and rectangular quadrature amplitude modulation $(I \times J$ QAM) under Nakagami- $m$ fading. The main contribution of this paper is to show that this alternative method can be used to reduce the computational complexity for detecting signals in the presence of fading.
\end{abstract}

\section{Introduction}

The growing need for improvement in capacity and performance of wireless communication systems has demanded high data transmission rates, in a scenario suitable to accommodate the ever-increasing multimedia traffic and new applications. In this context, spectrally efficient modulation schemes have attracted the attention of companies and academia. Quadrature amplitude modulation (QAM) is an attractive modulation scheme to achieve high transmission rates, without increasing the bandwidth of the wireless communication system.

Traditionally, the computation of the BEP of $M$-QAM has been carried out by calculating the symbol error probability or simply estimating it using lower or upper bounds [1]. Good approximations for the BEP of $M$-QAM subject to additive white Gaussian noise (AWGN) have been presented in [2,3] based on signal-space concepts and recursive algorithms. It is worth mentioning that although some approximate expressions give accurate error rates for high signal-to-noise ratio (SNR), the evaluation of the error rates using those expressions tends to deviate from their corresponding exact values when the SNR is low.

In spite of the attention devoted to the study of the BEP of QAM for an AWGN channel, a closed-form expression for the BEP of $M$-QAM for an AWGN channel has been derived only in 2002 [4].

Regarding the performance evaluation of QAM for a Rayleigh fading channel, the BEP has been addressed previously (e.g., [5-8]). In [5], the analytically derived BEP formula for 16-QAM and 64-QAM involves the computation of a definite integral (whose integrand is the product of the well-known $Q$-function and an exponential function) and yields results that match the curves obtained from simulation. Based on [9], Vitthaladevuni and Alouini obtained generic expressions for the BEP of hierarchical constellations 4/M-QAM [7].

In [10], Craig's method [11] for numerically computing the average error probability of two-dimensional $M$-ary signaling in AWGN is extended to give results to determine the average probability of symbol errors in slow Rayleigh fading. Dong et al. have determined in [10] the exact average 
symbol error probability for the 16-Star QAM subject to fading.

Concerning the performance evaluation of communication systems for Nakagami fading channels, the bit error rate performance of multiple-input multiple-output (MIMO) systems employing transmit diversity through orthogonal space time block coding (STBC) was addressed in [12]. Exact closed-form expressions were derived for the BEP of Gray-coded Pulse Amplitude Modulation (PAM) and QAM modulations when STBC was employed in the presence of Nakagami- $m$ fading. The analysis considered a singleinput single-output (SISO) channel approach, and the mathematical expressions for the BEP were obtained for integer values of the Nakagami fading parameter $m$.

The STBC coding was also considered in [13], where the authors applied the SISO equivalency of STBC in order to analyse its performance over nonselective Nakagami- $m$ fading channels in presence of spatial fading correlation.

In [14], the authors considered a more general framework of Nakagami- $m$ fading and derived an exact closedform expression for the Shannon capacity of STBC, setting the limit on the achievable average spectral efficiency by any adaptive modulation scheme employing STBC in Nakagami fading.

In [15], the authors considered the fact that a signal received from a fading channel is subject to a multiplicative distortion (MD) and to the usual additive noise-then, following a compensation of the MD, the signal fed to the detector may include only a single additive distortion term, which comprises the effects of the original additive noise, the MD, and the error in MD compensation. In [15], the probability density function of that single additive distortion was derived and used to obtain the error probability of some modulation schemes. A similar development was used in [16] —in which the authors have shown that the Rayleigh fading channel can be seen as an additive noise channel whose noise is modeled as the ratio between a Gaussian random variable (r.v.) and a Rayleigh r.v. The cumulative density function (CDF) of that noise has been derived in [17].

This paper extends the analysis of $[16,17]$ and presents a convenient method for obtaining the BEP for modulation schemes subject to Nakagami- $m$ fading. The methodology presented in this paper is used to obtain exact and closedform expressions for the BEP of $M$-PAM, $M$-QAM, and $I \times J$ QAM schemes subject to Nakagami- $m$ fading. It is important to mention that the closed-form BER expressions derived do not represent a contribution of the paper, because they are available in the literature and can be obtained using other approaches, and are derived to illustrate the proposed method.

The paper also shows how this approach can be used to reduce the number of operations required for detecting signals in the presence of fading. This can be seen as the main contribution of this paper, since a lower computational complexity leads to a more efficient power consumption and a smaller processing time. Considering handsets of cellular communication systems, low power consumption extends the battery lifetime, and small processing time enhances the performance for online applications such as video calling and web browsing.

The remaining of the paper is organized as follows. Section 2 presents the system model. Section 3 addresses the derivation of the CDF of the additive noise. In Section 4, the $\mathrm{CDF}$ is used to derive an exact expression for the BEP of $M$-QAM subject to Nakagami- $m$ fading. Section 5 is devoted to the derivation of an exact expression for the BEP of an $M$-PAM subject to Nakagami- $m$ fading, while Section 6 deals with the derivation of an exact expression for the BEP of the $I \times J$-QAM subject to Nakagami- $m$ fading. Section 7 presents simulation results and an analysis of the computational complexity for detecting signals in presence of fading. Concluding remarks are presented in Section 8.

\section{The System Model}

Consider the wireless system depicted in Figure 1, in which the transmitter uses $M$-ary modulation.

Assuming a frequency-nonselective slow-fading channel, the received signal $\mathbf{r}_{c}(t)$ can be expressed as

$$
\mathbf{r}_{c}(t)=\alpha e^{-j \phi} \mathbf{s}(t)+\mathbf{z}(t), \quad 0 \leq t \leq T,
$$

in which $\mathbf{s}(t)$ represents the transmitted signal, $\alpha$ is the fading amplitude, $\phi$ is the phase shift due to the channel, $\mathbf{z}(t)$ denotes the additive white Gaussian noise, and $T$ is the signaling interval.

The fading amplitude $\alpha$ is modeled as a Nakagami- $m$ r.v., whose probability density function (pdf) is expressed as

$$
p(\alpha)=\frac{2 m^{m} \alpha^{2 m-1}}{\Gamma(m) \Omega^{m}} e^{-m \alpha^{2} / \Omega} u(\alpha),
$$

in which $u(\cdot)$ is the unit step function, $\Gamma(\cdot)$ denotes de Gamma function, $\Omega$ is the average power of the transmitted signal envelope, and $m \geq 1 / 2$ is a parameter that controls the intensity of the fading. Large values of $m$ represent mild fading whereas small values correspond to severe fading. The special case of $m=1$ reduces the Nakagami- $m$ fading to Rayleigh fading.

The additive noise $\mathbf{z}(t)$ is modeled as a two-dimensional Gaussian r.v. with zero mean and variance $N_{0} / 2$ per dimension. Without loss of generality, a normalized fading power is considered; that is, $E\left[\alpha^{2}\right]=1$, in which $E[\cdot]$ is the expectation operator.

Assuming that the fading is sufficiently slow so that the phase shift $\phi$ can be estimated from the received signal without error, the receiver can perform the phase compensation (multiplication of $\mathbf{r}_{c}(t)$ by $e^{j \phi}$ ). Then, the resulting received signal $\mathbf{r}(t)$ can be expressed as

$$
\mathbf{r}(t)=\mathbf{r}_{c}(t) \cdot e^{j \phi}=\alpha \mathbf{s}(t)+\mathbf{z}(t) \cdot e^{j \phi}=\alpha \mathbf{s}(t)+\mathbf{n}(t) .
$$

It is important to note that $\mathbf{n}(t)=\mathbf{z}(t) \cdot e^{j \phi}$ is also a twodimensional Gaussian r.v. having zero mean and variance $N_{0} / 2$ per dimension. This follows from the fact that the error probability is unaffected by a rotation, since the pdf of the white Gaussian noise, $p_{N}(n)$, is spherically symmetric [18, page 247]. 


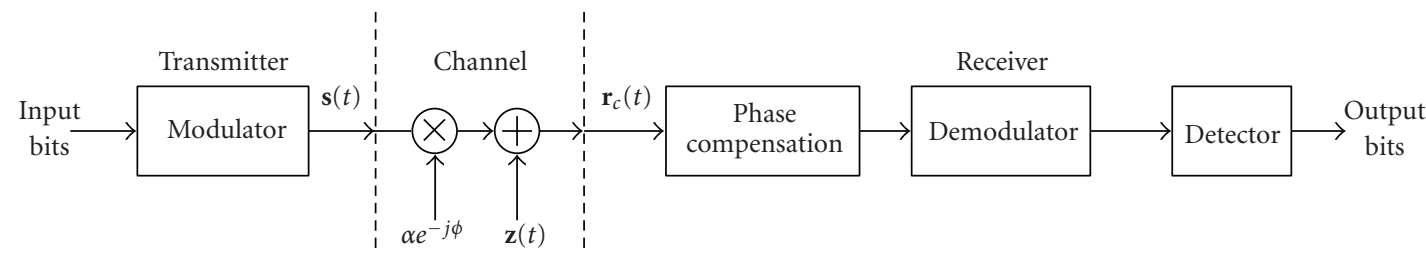

FIgURE 1: The system model.

The maximum a posteriori criterion [1] establishes that the optimum detector, on observing $\mathbf{r}(t)$, sets $\widehat{\mathbf{s}}(t)=\mathbf{s}_{k}(t)$ as the received symbol whenever the decision function

$$
\mathrm{P}\left(\mathbf{s}_{i}(t)\right) p_{r}\left(\mathbf{r}(t) \mid \mathbf{s}(t)=\mathbf{s}_{i}(t)\right), \quad i=0,1, \ldots, M-1
$$

is maximum for $i=k$, in which $p_{r}\left(\mathbf{r}(t) \mid \mathbf{s}(t)=\mathbf{s}_{i}(t)\right)$ is the conditional pdf of the observed signal $\mathbf{r}(t)$ given $\mathbf{s}(t)$ and $\mathrm{P}\left(\mathbf{s}_{i}(t)\right)$ is the a priori probability of the ith transmitted signal $\mathbf{s}_{i}(t)$.

Based on the maximum a posteriori criterion and considering equiprobable constellation symbols, two different strategies can be used for determining the most probable transmitted symbol from the noisy observation $\mathbf{r}(t)$. According to these strategies, two detectors can be defined:

(i) detector I (DI): compare $\mathbf{r}(t)$ with all the constellation symbols (multiplied by $\alpha$ ) and choose as the received symbol the closest one to $\mathbf{r}(t)$, that is, the one that minimizes the metric $\left|\mathbf{r}(t)-\alpha \mathbf{s}_{i}(t)\right|$;

(ii) detector II (DII): compare $\mathbf{r}(t) / \alpha$ with all the constellation symbols and choose as the received symbol the closest one to $\mathbf{r}(t) / \alpha$ that is, choose as the received symbol the one that minimizes the metric $\mid \mathbf{r}(t) / \alpha-$ $\mathbf{s}_{i}(t) \mid$.

For detector DII, after providing fading compensation (division of $\mathbf{r}(t)$ by $\alpha$ ), the channel can be seen as an additive noise channel because

$$
\begin{aligned}
\widehat{\mathbf{s}}(t) & =\arg \min _{\mathbf{s}_{i}(t)}\left|\frac{\alpha \mathbf{s}(t)+\mathbf{n}(t)}{\alpha}-\mathbf{s}_{i}(t)\right| \\
& =\arg \min _{\mathbf{s}_{i}(t)}\left|\mathbf{s}(t)+\mathbf{l}(t)-\mathbf{s}_{i}(t)\right|,
\end{aligned}
$$

in which $\mathbf{l}(t)=\mathbf{n}(t) / \alpha$ denotes the new additive noise obtained from the ratio between a Gaussian r.v. $N$ and a Nakagami- $m$ r.v. $\alpha$. This new additive noise is modeled by the r.v. $L=N / \alpha$, obtained from the random variables $N$ and $\alpha$, whose CDF is presented in the next section.

\section{Cumulative Density Function of $L=N / \alpha$}

The random variable $N$ with zero mean and variance $N_{0} / 2$ that models the white Gaussian noise has a pdf given by

$$
p_{N}(n)=\frac{1}{\sqrt{\pi N_{0}}} e^{-n^{2} / N_{0}}
$$

while the pdf of the random variable $\alpha$, which has Nakagami$m$ distribution, is given by (2).

According to [19], the probability density function of a random variable $L$ defined as the ratio between two random variables, $L=N / \alpha$, is given by

$$
p_{L}(l)=\int_{-\infty}^{\infty}|\alpha| p(l \alpha, \alpha) d \alpha .
$$

Since the variables $N$ and $\alpha$ are independent, the joint pdf $p(n, \alpha)$ can be written as

$$
p(n, \alpha)=p_{N}(n) p_{\alpha}(\alpha)=\frac{2 m^{m} \alpha^{2 m-1}}{\Gamma(m) \Omega^{m}} e^{-m \alpha^{2} / \Omega} \frac{e^{-n^{2} / N_{0}}}{\sqrt{\pi N_{0}}} u(\alpha),
$$

and the joint pdf $p(l \alpha, \alpha)$ can be obtained by substituting $n=$ $l \alpha$ into (8).

Therefore, the marginal pdf $p_{L}(l)$ can be obtained using (8) and (7) so that

$$
\begin{aligned}
p_{L}(l) & =\int_{-\infty}^{\infty} \frac{|\alpha| 2 m^{m} \alpha^{2 m-1}}{\Gamma(m) \Omega^{m}} \frac{e^{-m \alpha^{2} / \Omega}}{\sqrt{\pi N_{0}}} e^{-\left(l^{2} \alpha^{2}\right) / N_{0}} u(\alpha) d \alpha \\
& =\frac{2}{\sqrt{\pi N_{0}}} \frac{m^{m}}{\Gamma(m)} \frac{1}{\Omega^{m}} \int_{0}^{\infty} \alpha^{2 m} e^{-\alpha^{2}\left(m / \Omega+l^{2} / N_{0}\right)} d \alpha .
\end{aligned}
$$

It is observed in (9) that the integral has the form

$$
\int_{0}^{\infty} x^{2 m} e^{-\rho x^{2}} d x
$$

and the corresponding result is obtained as follows:

$$
\begin{aligned}
\int_{0}^{\infty} x^{2 m} e^{-\rho x^{2}} d x & =\int_{0}^{\infty} x^{2 m} e^{-(\sqrt{\rho} x)^{2}} d x \\
& =\int_{0}^{\infty}\left(\frac{u}{\sqrt{\rho}}\right)^{2 m} e^{-u^{2}} \frac{d u}{\sqrt{\rho}} \\
& =\frac{1}{2 \rho^{(m+1 / 2)}} \Gamma\left(m+\frac{1}{2}\right) .
\end{aligned}
$$

Thus,

$$
\begin{aligned}
& \int_{0}^{\infty} \alpha^{2 m} e^{-\alpha^{2}\left(m / \Omega+l^{2} / N_{0}\right)} d \alpha \\
& \quad=\frac{1}{2} \Gamma\left(m+\frac{1}{2}\right)\left(\frac{l^{2}}{N_{0}}+\frac{m}{\Omega}\right)^{-(m+1 / 2)} .
\end{aligned}
$$

Substituting the result from (9), it follows that the pdf of the random variable $L$, that represents the new noise 
(modeled as the ratio between two random variables), can be written as [20]

$$
p_{L}(l)=\frac{m^{m}}{\sqrt{\pi} \Omega^{m}} \frac{\Gamma(m+1 / 2)}{\sqrt{N_{o}} \Gamma(m)}\left(\frac{l^{2} \Omega+m N_{o}}{N_{o} \Omega}\right)^{-m-1 / 2} .
$$

The cumulative density function (CDF) as a function of $l, P_{L}(l)$, considering $\Omega=1$, is then obtained by calculating the integral

$$
\begin{aligned}
P_{L}(l)= & \int_{-\infty}^{l} p_{L}(x) d x=\frac{m^{m} N_{o}^{m}}{\sqrt{\pi}} \frac{\Gamma(m+1 / 2)}{\Gamma(m)} \\
& \times \int_{-\infty}^{l}\left(x^{2}+N_{o} m\right)^{-(2 m+1) / 2} d x .
\end{aligned}
$$

For noninteger values of $m$, the last improper integral in (14) could be expressed as [21]

$$
\int\left(x^{2}+a\right)^{-(2 m+1) / 2} d x=x \cdot{ }_{2} F_{1}\left(\frac{1}{2}, m+\frac{1}{2} ; \frac{3}{2} ;-\frac{x^{2}}{a}\right) a^{-(m+1 / 2)}
$$

in which ${ }_{2} F_{1}(a, b ; c ; x)$ is known as Gauss hypergeometric function. Is is worth to mention that another type of Lauricella hypergeometric function was used in [22] to determine the BEP of Nakagami-q (Hoyt) fading channels with spatial diversity.

The evaluation of the previous result as $x$ approximates $-\infty$ is carried out by considering the integral form of

$$
{ }_{2} F_{1}(a, b ; c ; x)=\frac{\Gamma(c)}{\Gamma(b) \Gamma(c-b)} \int_{0}^{1} \frac{t^{b-1}(1-t)^{c-b-1}}{(1-t x)^{a}} d t,
$$

and taking the limit

$$
\lim _{x \rightarrow-\infty} x \cdot 2 F_{1}\left(\frac{1}{2}, m+\frac{1}{2} ; \frac{3}{2} ;-\frac{x^{2}}{2}\right) .
$$

This limit can be calculated as follows:

$$
\begin{aligned}
\lim _{x \rightarrow-\infty} x & \cdot{ }_{2} F_{1}\left(\frac{1}{2}, m+\frac{1}{2} ; \frac{3}{2} ;-x^{2}\right) \\
= & \frac{\Gamma(3 / 2)}{\Gamma(m+1 / 2) \Gamma(1-m)} \int_{0}^{1} t^{m-1 / 2}(1-t)^{-m} \\
& \times \lim _{x \rightarrow-\infty} \frac{x}{\left(1+t x^{2}\right)^{1 / 2}} d t \\
= & -\frac{\Gamma(3 / 2)}{\Gamma(m+1 / 2) \Gamma(1-m)} \int_{0}^{1} t^{m-1}(1-t)^{-m} d t \\
= & -\frac{\Gamma(3 / 2) \mathrm{B}(m, 1-m)}{\Gamma(m+1 / 2) \Gamma(1-m)}, \quad 0<m<1,
\end{aligned}
$$

in which $\mathrm{B}(m, 1-m)$ is the beta function evaluated in $m$ and $1-m, 0<m<1[21]$.

The CDF of $L$ for $m>1$ may be obtained by considering

$$
B(x, y)=\frac{\Gamma(x) \Gamma(y)}{\Gamma(x+y)}, \quad \Gamma(x)=\frac{\Gamma(x+1)}{x}, \quad x<0 .
$$

Applying these results in the evaluation of the function $P_{L}(l)$ given in (14), it follows that

$$
\begin{aligned}
P_{L}(l)= & \frac{\Gamma(m+1 / 2)}{\sqrt{\pi} \Gamma(m)} \\
& \times\left\{\frac{l}{\sqrt{N_{o} m}} \times{ }_{2} F_{1}\left(\frac{1}{2}, m+\frac{1}{2} ; \frac{3}{2} ;-\frac{l^{2}}{N_{o} m}\right)\right. \\
& \left.+\frac{\Gamma(3 / 2) \mathrm{B}(m, 1-m)}{\Gamma(m+1 / 2) \Gamma(1-m)}\right\} .
\end{aligned}
$$

The expression of $P_{L}(l)$ for integer values of $m$ can be obtained by substituting the variable $x=\operatorname{tg}(\theta)$ in the integral of (15). The corresponding result is

$$
\int \frac{1}{\left(x^{2}+a\right)^{m+(1 / 2)}} d x=\frac{1}{a^{m}} \sum_{k=0}^{(m-1)} \frac{(-1)^{k} C_{m-1, k}}{(2 k+1)}\left[\frac{x}{\sqrt{x^{2}+a}}\right]^{2 k+1}
$$

in which

$$
C_{m-1, k}=\frac{(m-1) !}{k !(m-1-k) !} .
$$

By evaluating (21) as $x$ approximates $-\infty$ and $x=l$ and substituting the result in (14), the function $P_{L}(l)$ can be written as

$$
\begin{aligned}
P_{L}(l)= & \frac{\Gamma(m+1 / 2)}{\sqrt{\pi} \Gamma(m)} \times \sum_{k=0}^{(m-1)} \frac{(-1)^{k} C_{m-1, k}}{(2 k+1)} \\
& \times\left\{\left[\frac{l}{\sqrt{l^{2}+N_{0} m}}\right]^{2 k+1}+1\right\} .
\end{aligned}
$$

\section{BEP of $M$-QAM}

The CDF $P_{L}(l)$ can be used for computing the BEP of a square $M$-QAM system subject to Nakagami- $m$ fading using the result obtained in [9], in which Yoon and Cho used the bit mapping consistency of an $M$-QAM constellation under Gray coding to show that the BEP of a square $M$-QAM system subject to additive white Gaussian noise (AWGN), denoted by $P_{b}$, can be written as

$$
P_{b}=\frac{1}{\log _{2} \sqrt{M}} \sum_{k=1}^{\log _{2} \sqrt{M}} P_{b}(k)
$$

in which $P_{b}(k)$ is given by

$$
P_{b}(k)
$$$$
=\frac{1}{\sqrt{M}} \sum_{i=0}^{\left(1-2^{-k}\right) \sqrt{M}-1}\left\{w(i, k, M) \operatorname{erfc}\left((2 i+1) \sqrt{\frac{3 \log _{2} M \gamma}{2(M-1)}}\right)\right\},
$$ 
in which the weights $w(i, k, M)$ are

$$
w(i, k, M)=(-1)^{\left\lfloor 2^{k-1} / \sqrt{M}\right\rfloor} \cdot\left(2^{K-1}-\left\lfloor\frac{i \cdot 2^{k-1}}{\sqrt{M}}+\frac{1}{2}\right\rfloor\right),
$$

$\gamma=E_{b} / N_{0}$ denotes the signal-to-noise ratio per bit, $\lfloor x\rfloor$ denotes the largest integer smaller that $x, \operatorname{erfc}(\cdot)$ denotes the complementary error function and

$$
d=\sqrt{\frac{3 \log _{2}(M) E_{b}}{2(M-1)}}
$$

represents the minimum distance between two $M$-QAM symbol components.

It is important to note that the BEP of $M$-QAM modulation subject to AWGN is expressed in terms of a weighted sum of complementary error functions. The term $\operatorname{erfc}(\cdot)$ in (25) corresponds to twice the probability of the additive Gaussian noise exceeding $(2 i+1) \sqrt{\left(3 \log _{2} M \cdot E_{b}\right) / 2(M-1)}$. For non-Gaussian additive channels, the weights in (26) (which incorporates the effect on the BEP of the bit positions in a symbol with $\log _{2} M$ bits) can be used in conjunction with the cumulative density function (CDF) of the corresponding additive noise for determining the BEP of an $M$-QAM scheme.

Considering the Nakagami- $m$ fading channel, the probability that the intensity of the new additive noise $l(t)$ exceeds $\sqrt{3(2 i+1)^{2} \log _{2}(M) E_{b} /(M-1)}$ can be written as

$$
\begin{aligned}
\operatorname{Prob}\left(l \geq \sqrt{\frac{3(2 i+1)^{2} \log _{2}(M) E_{b}}{(M-1)}}\right) \\
\quad=1-P_{L}\left(\sqrt{\frac{3(2 i+1)^{2} \log _{2}(M) E_{b}}{(M-1)}}\right) .
\end{aligned}
$$

Using the previous result into the CDF obtained from (20), it follows that

$$
\begin{aligned}
& P_{L}\left(\sqrt{\frac{3(2 i+1)^{2} \log _{2}(M) E_{b}}{(M-1)}}\right) \\
& =\frac{\Gamma(m+1 / 2)}{\sqrt{\pi} \Gamma(m)} \sqrt{\frac{3(2 i+1)^{2} \log _{2}(M)}{m(M-1)} \frac{E_{b}}{N_{0}}} \\
& \quad \times{ }_{2} F_{1}\left(\frac{1}{2}, m+\frac{1}{2} ; \frac{3}{2} ;-\frac{3(2 i+1)^{2} \log _{2}(M)}{m(M-1)} \frac{E_{b}}{N_{0}}\right) \\
& \quad+\frac{\mathrm{B}(m, 1-m)}{2 \Gamma(m) \Gamma(1-m)} .
\end{aligned}
$$

Hence,

$$
\begin{aligned}
2 \operatorname{Prob}\left(l \geq \sqrt{\frac{3(2 i+1)^{2} \log _{2}(M) E_{b}}{(M-1)}}\right) \\
=2\left\{1-\frac{\Gamma(m+1 / 2)}{\Gamma(m) \sqrt{\pi}} \sqrt{\frac{3(2 i+1)^{2} \log _{2}(M)}{m(M-1)} \frac{E_{b}}{N_{0}}}\right. \\
\quad \times{ }_{2} F_{1}\left(\frac{1}{2}, m+\frac{1}{2} ; \frac{3}{2} ;-\frac{3(2 i+1)^{2} \log _{2}(M)}{m(M-1)} \frac{E_{b}}{N_{0}}\right) \\
\left.\quad-\frac{\mathrm{B}(m, 1-m)}{2 \Gamma(m) \Gamma(1-m)}\right\} .
\end{aligned}
$$

Therefore, the probability $P_{b}(k)$ of the square $M$-QAM can be written as

$$
\begin{aligned}
& P_{b}(k)=\frac{2}{\sqrt{M}} \sum_{i=0}^{\left(1-2^{-k}\right) \sqrt{M}-1} \\
& \times w(i, k, M)\left\{1-\frac{\Gamma(m+(1 / 2))}{\Gamma(m) \sqrt{\pi}} \sqrt{\frac{a_{i}(M) \gamma}{m}}\right. \\
& \quad \times{ }_{2} F_{1}\left(\frac{1}{2}, m+\frac{1}{2} ; \frac{3}{2} ;-\frac{a_{i}(M) \gamma}{m}\right) \\
& \\
&\left.\quad-\frac{\mathrm{B}(m, 1-m)}{2 \Gamma(m) \Gamma(1-m)}\right\},
\end{aligned}
$$

in which

$$
a_{i}(M)=\frac{3(2 i+1)^{2} \log _{2}(M)}{M-1} .
$$

Considering the representation of the beta function from (19), the probability $P_{b}(k)$ can be written as

$$
\begin{aligned}
P_{b}(k)= & \frac{2}{\sqrt{M}} \sum_{i=0}^{\left(1-2^{-k}\right) \sqrt{M}-1} \\
& \times w(i, k, M)\left\{\frac{1}{2}-\frac{\Gamma(m+1 / 2)}{\Gamma(m) \sqrt{\pi}} \sqrt{\frac{a_{i}(M) \gamma}{m}}\right. \\
& \left.\quad \times{ }_{2} F_{1}\left(\frac{1}{2}, m+\frac{1}{2} ; \frac{3}{2} ;-\frac{a_{i}(M) \gamma}{m}\right)\right\} .
\end{aligned}
$$


For integer values of $m, P_{b}(k)$ is obtained using the CDF $P_{L}(l)$ given in (23)

$$
\begin{aligned}
P_{b}(k)= & \frac{2}{\sqrt{M}} \sum_{i=0}^{\left(1-2^{-k}\right) \sqrt{M}-1} \\
& \times w(i, k, M)\left\{1-\frac{\Gamma(m+1 / 2)}{\sqrt{\pi} \Gamma(m)} \sum_{k=0}^{(m-1)} \frac{(-1)^{k} C_{m-1, k}}{(2 k+1)}\right. \\
& \left.\times\left[\left(\frac{a_{i}(M) \gamma}{a_{i}(M) \gamma+m}\right)^{k+1 / 2}+1\right]\right\} .
\end{aligned}
$$

\section{BEP of the $M$-PAM Scheme}

The signal waveforms of an $M$-ary pulse amplitude modulation can be expressed as

$$
s(t)=A_{I} \cos \left(2 \pi f_{c} t\right), \quad 0 \leq t<T
$$

in which $A_{I}$ is the amplitude of the in-phase component, $f_{c}$ is the carrier frequency and $T$ is the symbol interval. In an $M$-PAM scheme, $\log _{2} M$ bits are used to select the amplitude $A_{I}$ from the set $\{ \pm d, \pm 3 d, \ldots, \pm(M-1) d\}$, in which $2 d$ is the minimum distance between two distinct symbols, with

$$
d=\sqrt{\frac{3 \log _{2} M \cdot E_{b}}{\left(M^{2}-1\right)}}
$$

in which $E_{b}$ is the bit energy. The received PAM signal can be demodulated coherently.

In [4], a closed-form expression for the BEP of $M$ PAM under additive white Gaussian noise (AWGN) has been derived. In the following, results presented by Cho and Yoon are used to obtain a closed-form expression for the BEP of $M$-PAM subject to Nakagami- $m$ fading.

Based on the consistency of the bit mapping of a Gray coded signal constellation, Cho and Yoon have derived in [4] an expression for the BEP of the square $M$-PAM scheme for an AWGN channel. It is given by

$$
P_{b}=\frac{1}{\log _{2}(M)} \sum_{k=1}^{\log _{2}(M)} P_{b}(k)
$$

with the probability $P_{b}(k)$ written as

$$
\begin{aligned}
P_{b}(k)=\sum_{i=0}^{\left(1-2^{-k}\right) M-1}\{ & \frac{w(i, k, M)}{M} \\
& \left.\times \operatorname{erfc}\left((2 i+1) \sqrt{\frac{3 \log _{2} M \gamma}{\left(M^{2}-1\right)}}\right)\right\},
\end{aligned}
$$

where

$$
w(i, k, M)=(-1)^{\left\lfloor 2^{k-1} / M\right\rfloor} \cdot\left(2^{k-1}-\left\lfloor\frac{i \cdot 2^{k-1}}{M}+\frac{1}{2}\right\rfloor\right) .
$$

Using the CDFs given in (20) and (23), the probability $P_{b}(k)$ can be expressed as

$$
\begin{aligned}
P_{b}(k)= & \frac{2}{M} \sum_{i=0}^{\left(1-2^{-k}\right) M-1} w(i, k, M) \\
& \times\left\{1-\frac{\Gamma(m+1 / 2)}{\Gamma(m) \sqrt{\pi}} \sqrt{\frac{a_{i}(M) \gamma}{m}}\right. \\
& \times{ }_{2} F_{1}\left(\frac{1}{2}, m+\frac{1}{2} ; \frac{3}{2} ;-\frac{a_{i}(M) \gamma}{m}\right) \\
& \left.-\frac{\mathrm{B}(m, 1-m)}{2 \Gamma(m) \Gamma(1-m)}\right\} .
\end{aligned}
$$

Using the beta function expression from (19), the probability $P_{b}(k)$ can be written as

$$
\begin{aligned}
P_{b}(k)=\frac{2}{M} \sum_{i=0}^{\left(1-2^{-k}\right) M-1} w(i, k, M) \\
\times\left\{\frac{1}{2}-\frac{\Gamma(m+1 / 2)}{\Gamma(m) \sqrt{\pi}} \sqrt{\frac{a_{i}(M) \gamma}{m}}\right. \\
\left.\quad \times{ }_{2} F_{1}\left(\frac{1}{2}, m+\frac{1}{2} ; \frac{3}{2} ;-\frac{a_{i}(M) \gamma}{m}\right)\right\} .
\end{aligned}
$$

For integer values of $m, P_{b}(k)$ may be written as

$$
\begin{aligned}
P_{b}(k)=\frac{2}{M} & \sum_{i=0}^{\left(1-2^{-k}\right) M-1} w(i, k, M) \\
\times & \left\{1-\frac{\Gamma(m+1 / 2)}{\sqrt{\pi} \Gamma(m)} \sum_{k=0}^{(m-1)} \frac{(-1)^{k} C_{m-1, k}}{(2 k+1)}\right. \\
\times & {\left.\left[\left(\frac{a_{i}(M) \gamma}{a_{i}(M) \gamma+m}\right)^{k+1 / 2}+1\right]\right\}, }
\end{aligned}
$$

in which the term $a_{i}(M)$ is given by

$$
a_{i}(M)=\frac{6(2 i+1)^{2}}{\left(M^{2}-1\right)} \log _{2} M
$$

and the signal-to-noise ratio per bit $\gamma=E_{b} / N_{0}$.

\section{BEP of $I \times J$-QAM}

In an arbitrary rectangular $I \times J$-QAM scheme, the signal waveforms consist of two independently amplitudemodulated carriers in quadrature, that can be expressed as

$$
s(t)=A_{I} \cos \left(2 \pi f_{c} t\right)-A_{J} \sin \left(2 \pi f_{c} t\right), \quad 0 \leq t<T,
$$

in which $A_{I}$ and $A_{J}$ are the amplitudes of in-phase and quadrature components, respectively, $f_{c}$ is the carrier frequency and $T$ is the symbol interval. In an arbitrary $I \times J$ QAM scheme, $\log _{2}(I \cdot J)$ information bits are mapped into 
a two-dimensional constellation symbol using the Gray code. Among the $\log _{2}(I \cdot J)$ bits, $\log _{2} I$ bits are mapped into the in-phase component, the amplitude $A_{I}$ of which is selected from the set $\left\{ \pm d_{I}, \pm 3 d_{I}, \ldots, \pm(I-1) d_{I}\right\}$, in which $2 d_{I}$ is the minimum distance between the in-phase components of two distinct symbols. Similarly, $\log _{2} J$ bits are mapped into the quadrature component, the amplitude $A_{J}$ of which is selected from the set $\left\{ \pm d_{J}, \pm 3 d_{J}, \ldots, \pm(I-1) d_{J}\right\}$, in which $2 d_{J}$ is the minimum distance between the quadrature components of two distinct symbols. The demodulation of the received $I \times J$ QAM signal can be achieved by performing two parallel $M$ PAM demodulations.

For this modulation scheme, the function $\operatorname{erfc}(\cdot)$ in the expression of $P_{b}(k)$ in the $M$-PAM modulation must be substituted by

$$
\begin{aligned}
& \text { Prob }\left(l \geq \sqrt{\frac{6(2 i+1)^{2} \log _{2}(I \cdot J) E_{b}}{I^{2}+J^{2}-2}}\right) \\
& =2\left\{1-\frac{\Gamma(m+1 / 2)}{\Gamma(m) \sqrt{\pi}} \sqrt{\frac{6(2 i+1)^{2} \log _{2}(I \cdot J)}{m\left(I^{2}+J^{2}-2\right)} \gamma}\right. \\
& \quad \times{ }_{2} F_{1}\left(\frac{1}{2}, m+\frac{1}{2} ; \frac{3}{2} ;-\frac{6(2 i+1)^{2} \log _{2}(I \cdot J)}{m\left(I^{2}+J^{2}-2\right)} \gamma\right) \\
& \left.-\frac{\mathrm{B}(m, 1-m)}{2 \Gamma(m) \Gamma(1-m)}\right\} .
\end{aligned}
$$

The corresponding BEP can be written as

$$
P_{b}=\frac{1}{\log _{2}(I \cdot J)}\left(\sum_{k=1}^{\log _{2} I} P_{I}(k)+\sum_{l=1}^{\log _{2} J} P_{J}(l)\right)
$$

in which $P_{I}(k)$ and $P_{J}(k)$ are given by

$$
\begin{aligned}
& P_{I}(k)=\frac{2}{I} \sum_{i=0}^{\left(1-2^{-k}\right) I-1} w(i, k, I) \\
& \times\left\{1-\frac{\Gamma(m+1 / 2)}{\Gamma(m) \sqrt{\pi}} \sqrt{\frac{\gamma a_{i}(I, J)}{m}}\right. \\
& \times{ }_{2} F_{1}\left(\frac{1}{2}, m+\frac{1}{2} ; \frac{3}{2} ;-\frac{\gamma a_{i}(I, J)}{m}\right) \\
&\left.\quad-\frac{\mathrm{B}(m, 1-m)}{2 \Gamma(m) \Gamma(1-m)}\right\}, \\
& P_{J}(k)=\frac{2}{J} \sum_{j=0}^{\left(1-2^{-k}\right) J-1} w(j, k, J) \\
& \times\left\{1-\frac{\Gamma(m+1 / 2)}{\Gamma(m) \sqrt{\pi}}\right.
\end{aligned}
$$

$$
\begin{aligned}
& \times{\sqrt{\frac{\gamma a_{j}(I, J)}{m}}}_{2} F_{1}\left(\frac{1}{2}, m+\frac{1}{2} ; \frac{3}{2} ;-\frac{\gamma a_{j}(I, J)}{m}\right) \\
& \left.-\frac{\mathrm{B}(m, 1-m)}{2 \Gamma(m) \Gamma(1-m)}\right\},
\end{aligned}
$$

in which

$$
a_{j}(I, J)=\frac{6(2 j+1)^{2} \log _{2}(I \cdot J)}{I^{2}+J^{2}-2} .
$$

Using the beta function representation from $(19), P_{I}(k)$ and $P_{J}(k)$ can be simplified as

$$
\begin{aligned}
& P_{I}(k)=\frac{2}{I} \sum_{i=0}^{\left(1-2^{-k}\right) I-1} w(i, k, I) \\
& \times\left\{\frac{1}{2}-\frac{\Gamma(m+1 / 2)}{\Gamma(m) \sqrt{\pi}} \sqrt{\frac{\gamma a_{i}(I, J)}{m}}\right. \\
& \left.\quad \times{ }_{2} F_{1}\left(\frac{1}{2}, m+\frac{1}{2} ; \frac{3}{2} ;-\frac{\gamma a_{i}(I, J)}{m}\right)\right\}, \\
& P_{J}(k)=\frac{2}{J} \sum_{j=0}^{\left(1-2^{-k}\right) J-1} w(j, k, J) \\
& \times\left\{\frac{1}{2}-\frac{\Gamma(m+(1 / 2))}{\Gamma(m) \sqrt{\pi}} \sqrt{\frac{\gamma a_{j}(I, J)}{m}}\right. \\
& \left.\times{ }_{2} F_{1}\left(\frac{1}{2}, m+\frac{1}{2} ; \frac{3}{2} ;-\frac{\gamma a_{j}(I, J)}{m}\right)\right\} .
\end{aligned}
$$

The expressions for the weights $w(i, k, I)$ and $w(j, k, J)$ are given, respectively, by [9]

$$
\begin{aligned}
& w(i, k, I)=(-1)^{\left\lfloor 2^{k-1 / \sqrt{I}\rfloor}\right.} \cdot\left(2^{k-1}-\left\lfloor\frac{i \cdot 2^{k-1}}{\sqrt{I}}+\frac{1}{2}\right\rfloor\right), \\
& w(j, l, J)=(-1)^{\left\lfloor j 2^{l-1 / \sqrt{J}\rfloor}\right.} \cdot\left(2^{l-1}-\left\lfloor\frac{j \cdot 2^{l-1}}{\sqrt{J}}+\frac{1}{2}\right\rfloor\right) .
\end{aligned}
$$

It is interesting to point out that the expressions derived in the previous sections, written in terms of the hypergeometric function, gamma and beta functions, converge to those results obtained in the paper [23] when the parameter $m$ of the Nakagami- $m$ distribution is unity, corresponding to Rayleigh fading.

\section{Results}

This section presents numerical and Monte Carlo simulations results for the BEP of various modulations schemes subject to Nakagami- $m$ fading. A computational complexity of the proposed method is also carried out in Section 7.4 


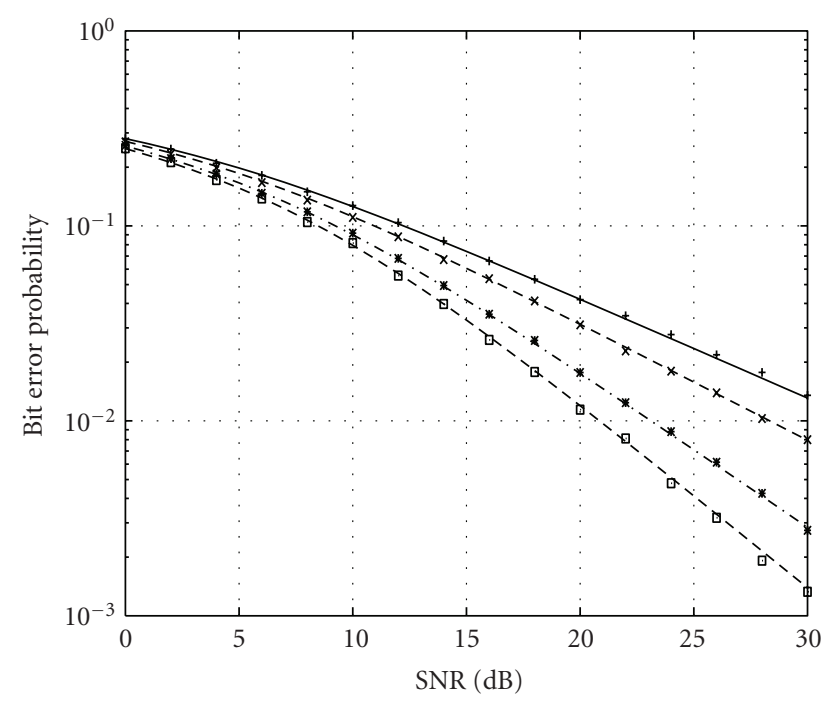

$\begin{aligned}-m=0.51 \text { (analytical) } & +m=0.51 \text { (simulation) } \\ ---m=0.6 \text { (analytical) } & \times m=0.6 \text { (simulation) } \\ ---m=0.8 \text { (analytical) } & \text { * } m=0.8 \text { (simulation) } \\ ---m=0.95 \text { (analytical) } & \text { } m=0.95 \text { (simulation) }\end{aligned}$

FIgURE 2: BEP for 64-QAM subject to Nakagami- $m$ fading for different values of $m, 0.5<m<1$.

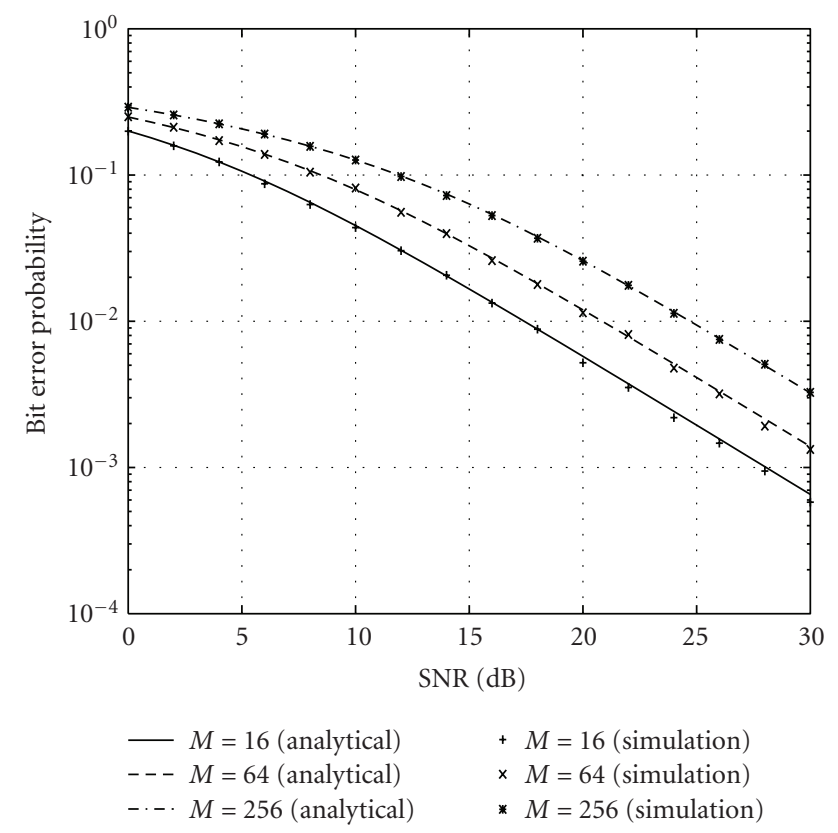

FIgURe 3: BEP for $M$-QAM subject to Nakagami- $m$ fading for $m=$ 0.95 and different values of $M$.

7.1. M-QAM Modulation Scheme. BEP curves for 64-QAM modulation scheme obtained from (33) are presented in Figure 2. They were obtained for different values of parameter $m$ of the Nakagami- $m$ distribution. For a fixed SNR, it is observed that the BEP increases as $m$ decreases to 0.51 . For the 64-QAM scheme it is observed that about $5 \mathrm{~dB}$ must be invested in the channel SNR in order to maintain a BEP of $10^{-2}$ if the parameter $m$ changes from 0.80 to 0.60 . It is also
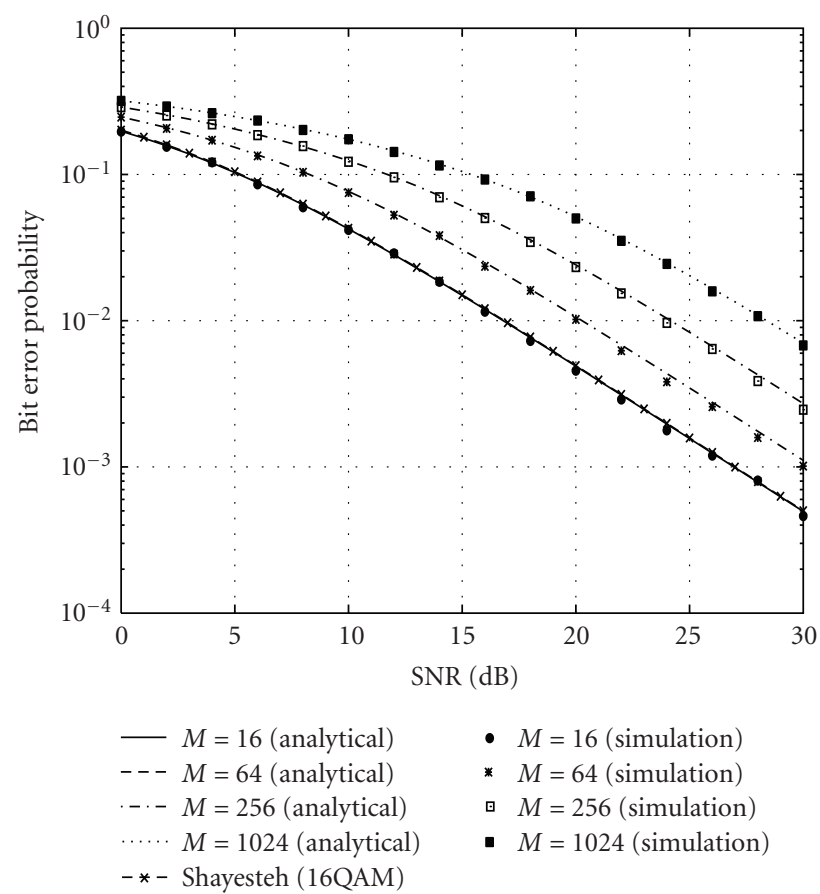

Figure 4: BEP for $M$-QAM subject to Nakagami- $m$ fading for $m=$ 1 and different values of $M$.

observed that for a $30 \mathrm{~dB}$ channel SNR, the BEP for $m=0.51$ is about ten times higher than that one for $m=0.95$.

In Figure 3 BEP curves are grouped for $m$ fixed and different values of $M$. It is observed in Figure 3 that the 256QAM system is more susceptible to fading than the 16-QAM scheme. It is an expected result because, for a given SNR, the Euclidean distance between the components of two distinct symbols in 256-QAM constellation is smaller than the one for 16-QAM.

It is worth mentioning that Nakagami- $m$ fading with $m=1$ corresponds to Rayleigh fading. When $m=1$ and $M=16$, the results obtained in this paper for the 16-QAM modulation agree with the results obtained by Shayesteh and Aghamohammadi in [15] for Rayleigh fading by using

$$
\begin{aligned}
P_{16-\text { QAM }, \text { Shay }}= & \frac{1}{2}+\frac{1}{8} \Theta(\gamma, 10) \\
& -\frac{1}{4} \Theta(\gamma, 0.4) \cdot\left\{1+\frac{1}{\pi} \operatorname{tg}^{-1}(3 \Theta(\gamma, 0.4))\right\} \\
& -\frac{1}{4} \Theta(\gamma, 3.6) \cdot\left\{1+\frac{1}{\pi} \operatorname{tg}^{-1}\left(\frac{1}{3} \Theta(\gamma, 3.6)\right)\right\},
\end{aligned}
$$

in which $\gamma=E_{b} / N_{o}$ and $\Theta(\gamma, a)=\sqrt{(a \cdot \gamma) /(a \cdot \gamma+1)}$.

Results obtained for $m=1$ in Figure 4 show that the modulation schemes with larger constellations are more susceptible to Rayleigh fading, which is usual in urban environments. In spite of that susceptibility, schemes with larger constellations are widely used when higher bit rates are needed. From Figure 4 it is observed that the 16-QAM 


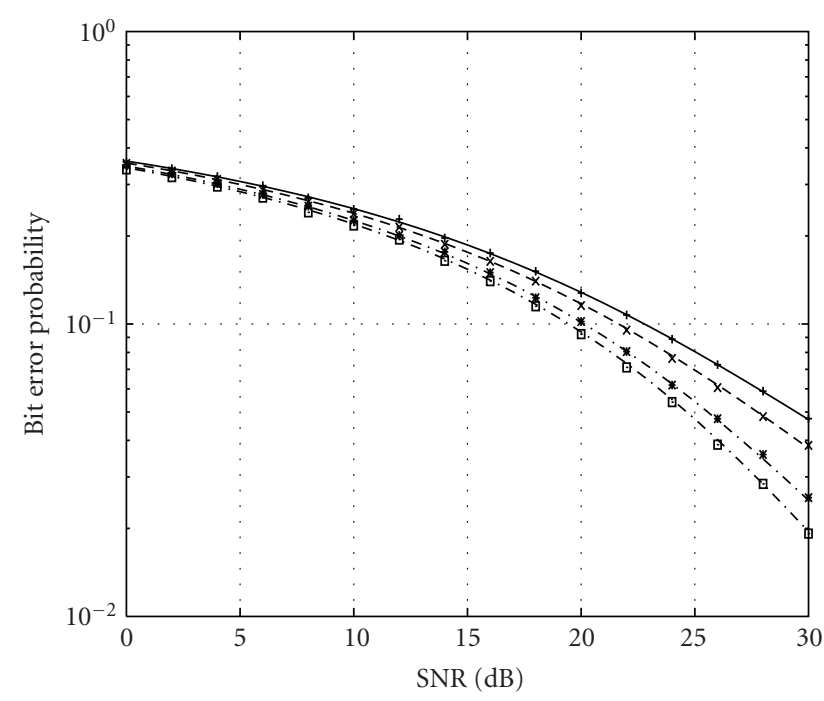

$\begin{array}{rlrl}-m & =0.51 \text { (analytical) } & +m=0.51 \text { (simulation) } \\ ---m=0.6 \text { (analytical) } & \times m=0.6 \text { (simulation) } \\ -\cdot-m=0.8 \text { (analytical) } & \text { * } m=0.8 \text { (simulation) } \\ -\cdot-m=0.95 \text { (analytical) } & \text { 口 } m=0.95 \text { (simulation) }\end{array}$

Figure 5: BEP for 64-PAM subject to Nakagami- $m$ fading for different values of $m$.

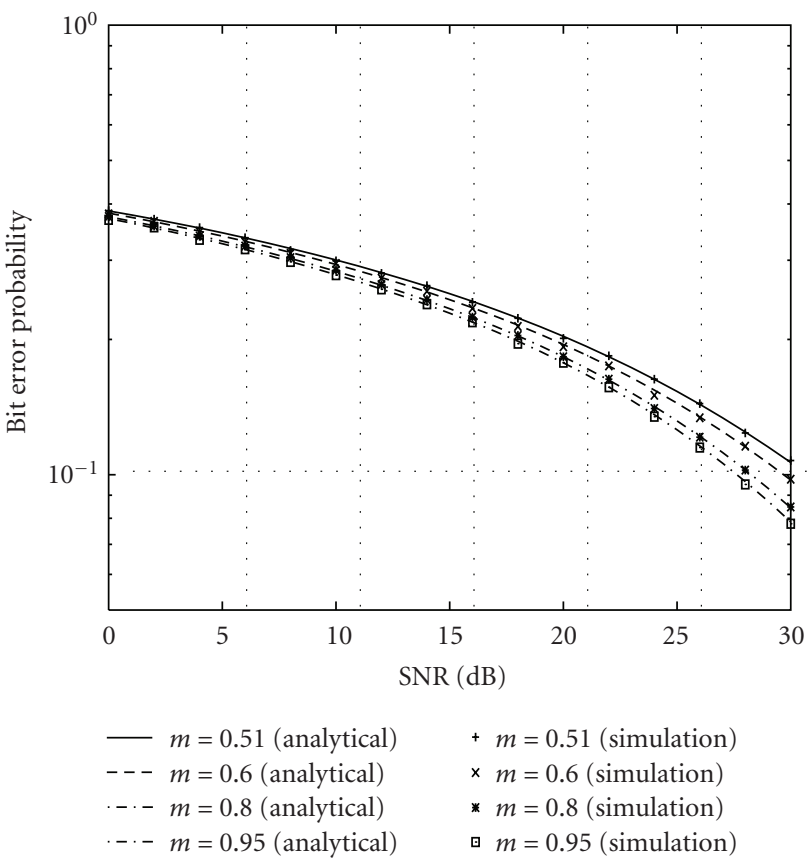

Figure 6: BEP for 256-PAM subject to Nakagami- $m$ fading for different values of $m$.

curve corroborates Shayesteh and Aghamohammadi results obtained from (54).

7.2. M-PAM Modulation Scheme. BEP curves for the 64PAM modulation scheme obtained from (37) and (41) are presented in Figure 5. The curves are presented for different values of the parameter $m$ of Nakagami- $m$ distribution. For

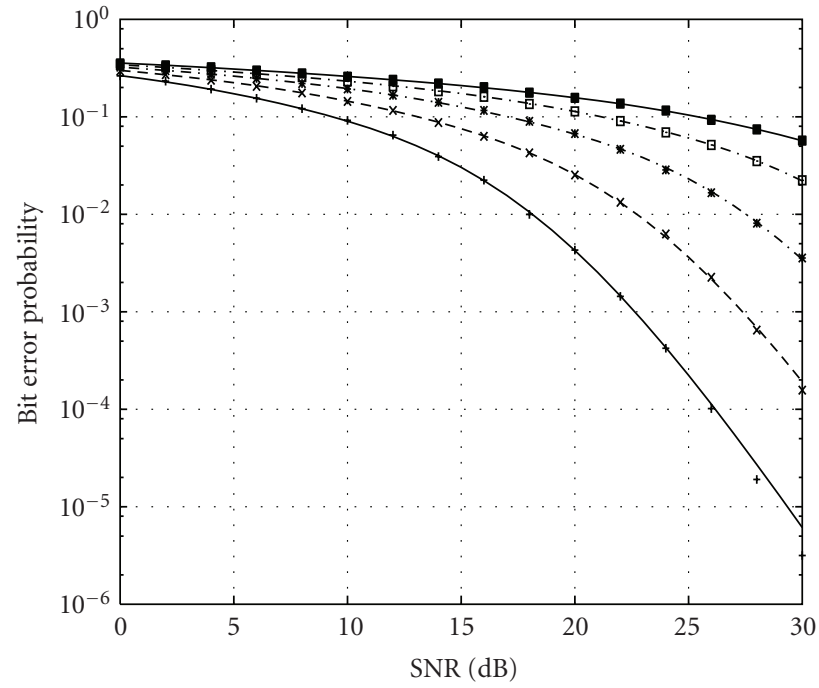

$$
\begin{aligned}
& \text { - } M=16 \text { (analytical) } \\
& +M=16 \text { (simulation) } \\
& \text { - - } M=32 \text { (analytical) } \\
& \text {-. - } M=64 \text { (analytical) } \\
& \text {-. } M=128 \text { (analytical) } \\
& \text {-. }-M=256 \text { (analytical) } \\
& \times M=32 \text { (simulation) } \\
& \text { * } M=64 \text { (simulation) } \\
& \text { - } M=128 \text { (simulation) } \\
& \text { - } M=256 \text { (simulation) }
\end{aligned}
$$

Figure 7: BEP for $M$-PAM subject to Nakagami- $m$ fading for $m=$ 3.50 and different values of $M$.

a fixed SNR, it is observed that the bit error probability increases as $m$ decreases to 0.51 .

Results for 256-PAM subject to Nakagami- $m$ fading are presented in Figure 6. An SNR increase of about $3 \mathrm{~dB}$ in the 256-PAM scheme is needed to preserve the BEP level, $P_{b}=$ 0.1 , when $m$ decreases from 0.95 to 0.51 .

BEP curves for $M$-PAM subject to Nakagami- $m$ fading for $m=3.50$ are presented in Figure 7. One can observe that the BEP in the 64-PAM scheme varies more, for a fixed SNR, when compared to the 256-PAM scheme.

7.3. $I \times J$-QAM Modulation Scheme. BEP curves for the $I \times J$ QAM constellation are obtained from (46) to (53).

Results for the $16 \times 32$-QAM scheme for $m$ ranging from 0.51 to 0.80 and from 1.25 to 1.55 are presented, respectively, in Figures 8 and 9. Comparing Figures 8 and 9, for the same constellation dimension, it is possible to notice the influence of the Nakagami- $m$ fading intensity, controlled by $m$, on the bit error probability. The BEP increases as parameter $m$ decreases.

It is observed from Figure 10 that the higher the constellation dimension the higher the BEP. It is observed that to keep the BEP in $10^{-2}$, an increase of $7.5 \mathrm{~dB}$ on the SNR must be provided when the $4 \times 8$-QAM scheme is substituted by the $16 \times 32$-QAM one to increase the transmission rate.

7.4. Computational Complexity Analysis. This section presents a computational complexity analysis for signal detection in the presence of fading by using the approach in which the communication channel is seen as an additive channel. The analysis is performed for two types of detectors, 


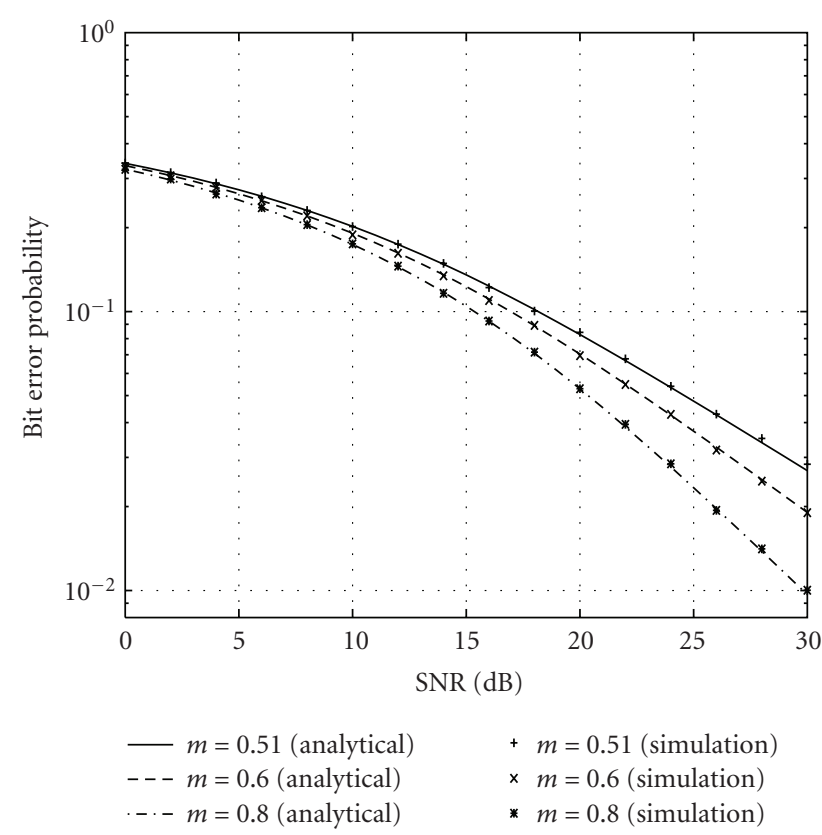

FIgURE 8: BEP for $16 \times 32$-QAM subject to Nakagami- $m$ fading for different values of $m$, ranging from 0.51 to 0.80 .

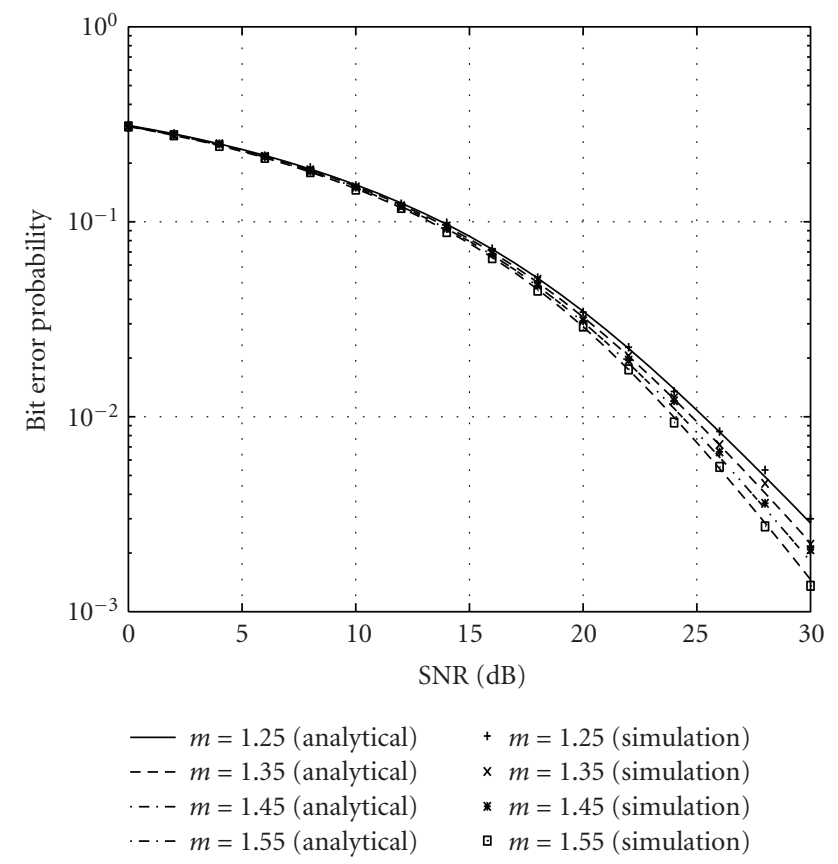

Figure 9: BEP for $16 \times 32$-QAM subject to Nakagami fading for different values of $m$, ranging from 1.25 to 1.55 .

referred to as Detector DI and Detector DII, as described in Section 2.

7.4.1. Detector DI. Considering an arbitrary $M$-ary modulation scheme, after receiving $\mathbf{r}(t)$, the detector DI computes the metrics $\left|\mathbf{r}(t)-\alpha \mathbf{s}_{i}(t)\right|$ for $i=0,1,2, M-1$ and compares them to obtain the minimum value.
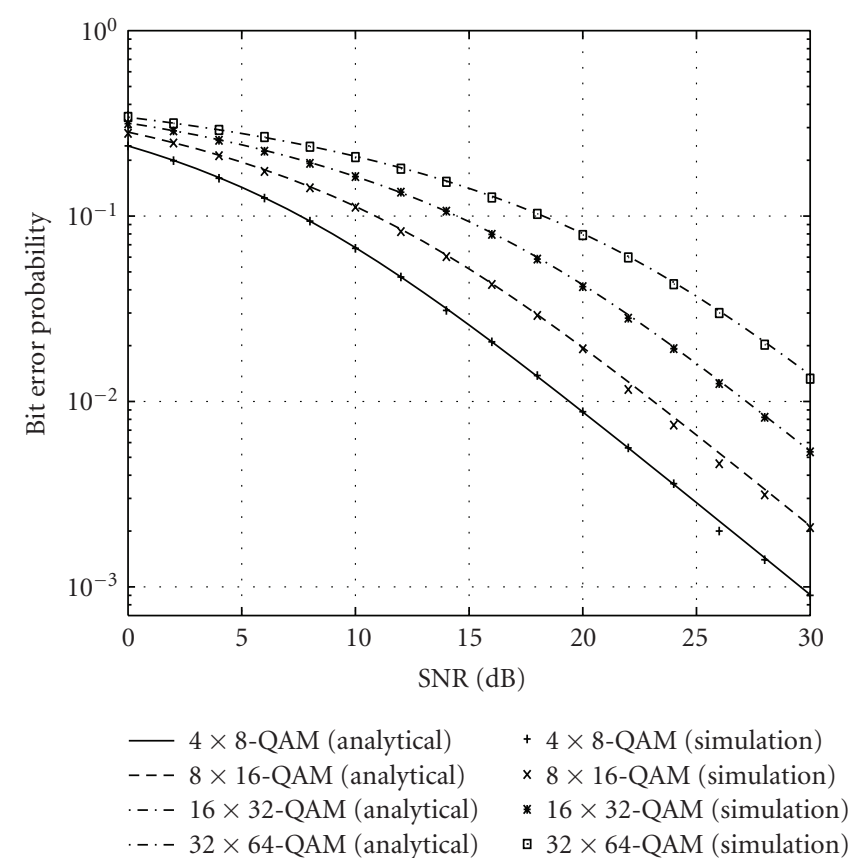

FIGURE 10: BEP for $I \times J$-QAM subject to Rayleigh fading $(m=1)$ and different values of $I$ and $J$.

TABLe 1: Number of real operations required by detector DI to obtain the estimate $\tilde{\mathbf{s}}(t)$ of the transmitted symbol $\mathbf{s}(t)$ based on the noisy observation $\mathbf{r}(t)$ considering $M=4$.

\begin{tabular}{lc}
\hline Operation & Number \\
\hline multiplication & 8 \\
subtraction & 8 \\
modulus & 4 \\
comparison & 3 \\
\hline
\end{tabular}

For each $\mathbf{s}_{i}(t)$, the detector performs:

(i) one real-complex multiplication: $\alpha \mathbf{s}_{i}(t)$, that is, two real multiplications,

(ii) one complex subtraction: $\mathbf{r}(t)-\alpha \mathbf{s}_{i}(t)$, that is, two real subtraction,

(iii) one modulus operation.

Since there are $M$ constellation symbols $\mathbf{s}_{i}$, the detector performs $2 M$ multiplications, $2 M$ subtractions and $M$ modulus operations. At this point, the detector has $M$ values of metric. Then, $M-1$ comparisons are performed to find the minimum value which corresponds to the most probable $\mathbf{s}_{i}(t)$. Table 1 summarizes the total number of operations required for detector DI, considering $M=4$.

7.4.2. Detector DII. After receiving $\mathbf{r}(t)$, detector DII computes the metrics $\left|\mathbf{r}(t) / \alpha-\mathbf{s}_{i}(t)\right|$ for $i=0,1,2, M-1$ and compares them to obtain the minimum value. It is important to note that the detector calculates $\mathbf{r}(t) / \alpha$ only once. This corresponds to one complex-real division (two real divisions). 
TABLE 2: Number of real operations required by detector DII to obtain the estimate $\widetilde{\mathbf{s}}(t)$ of the transmitted symbol $\mathbf{s}(t)$ based on the noisy observation $\mathbf{r}(t)$ considering $M=4$.

\begin{tabular}{lc}
\hline Operation & Number \\
\hline division & 2 \\
subtraction & 8 \\
modulus & 4 \\
comparison & 3 \\
\hline
\end{tabular}

TABLE 3: Number of real multiplications required by detector DI and the number of real divisions required by detector DII to obtain the estimate $\widetilde{\mathbf{s}}(t)$ of the transmitted symbol $\mathbf{s}(t)$ based on the noisy observation $\mathbf{r}(t)$ as a function of $M$.

\begin{tabular}{lcc}
\hline & $\begin{array}{c}\text { Detector DI } \\
\text { multiplications }\end{array}$ & $\begin{array}{c}\text { Detector DII } \\
\text { divisions }\end{array}$ \\
\hline 4-QAM & 8 & 2 \\
16-QAM & 32 & 2 \\
64-QAM & 128 & 2 \\
256-QAM & 512 & 2 \\
\hline 16-PAM & 32 & 2 \\
32-PAM & 64 & 2 \\
64-PAM & 128 & 2 \\
128-PAM & 256 & 2 \\
\hline 8×16-QAM & 256 & 2 \\
16×32-QAM & 1024 & 2 \\
\hline
\end{tabular}

After that, for each $\mathbf{s}_{i}(t)$ the detector performs:

(i) one complex subtraction $\left(\mathbf{r}(t) / \alpha-\mathbf{s}_{i}(t)\right)$, that is, two real subtractions,

(ii) one modulus operation.

Finally, the detector DII has $M$ values of metric. Therefore, DII must perform $M-1$ comparisons to find the minimum value and consequently the most probable $\boldsymbol{s}_{i}(t)$. Table 2 summarizes the total number of operations required for detector DII considering $M=4$.

7.4.3. Performance Comparison. Tables 1 and 2 show that both detection schemes perform the same number of subtractions, comparisons, and moduli. The basic difference is that detector DI requires $2 M$ multiplications while detector DII requires only 2 divisions.

According to Knuth [24], a multiplication or a division of two $n$-bit numbers can be accomplished in $O(n \log n \log \log n)$ steps. Current implementations of those operations can actually be done using the same number of clock cycles. Thus, it is reasonable to assign the same complexity for both multiplication and division. Based on those statements, one can conclude that detector DII has a lower complexity when compared to that of detector DI. The proposed approach becomes more attractive for higher order modulation schemes. For instance, detector DII requires only 2 divisions for detecting a 256-QAM symbol while detector DI requires 512 multiplications to perform the same task.

\section{Conclusion}

This paper presented an alternative method to obtain the BEP of modulation schemes subject to Nakagami- $m$ fading. In the approach, the Nakagami- $m$ fading channel is seen as an additive noise channel whose noise is modeled as the ratio between a Gaussian random variable and a Nakagami$m$ random variable. Exact and closed-form expressions were obtained for the BEP of the modulation schemes square $M$-QAM, $M$-PAM, and rectangular $I \times J$-QAM, subject to Nakagami- $m$ fading. The mathematical expressions are written in terms of the Hypergeometric function. The main contribution of this paper is to show that this alternative method can be used to reduce the computational complexity for detecting signals in the presence of fading.

\section{References}

[1] J. G. Proakis, Digital Communications, McGraw-Hill, New York, NY, USA, 3rd edition, 1995.

[2] J. Lu, K. B. Letaief, J. C.-I. Chuang, and M. L. Liou, "M-PSK and $M$-QAM BER computation using signal-space concepts," IEEE Transactions on Communications, vol. 47, no. 2, pp. 181184, 1999.

[3] L.-L. Yang and L. Hanzo, "Recursive algorithm for the error probability evaluation of M-QAM," IEEE Communications Letters, vol. 4, no. 10, pp. 304-306, 2000.

[4] K. Cho and D. Yoon, "On the general BER expression of one- and two-dimensional amplitude modulations," IEEE Transactions on Communications, vol. 50, no. 7, pp. 10741080, 2002.

[5] P.-M. Fortune, L. Hanzo, and R. Steele, "On the computation of 16-QAM and 64-QAM performance in Rayleigh-fading channels," IEICE Transactions on Communications, vol. E75-B, no. 6, pp. 466-475, 1992.

[6] L. Hanzo, R. Steele, and P. Fortune, "A subband coding, $\mathrm{BCH}$ coding, and 16-QAM system for mobile radio speech communications," IEEE Transactions on Vehicular Technology, vol. 39, no. 4, pp. 327-339, 1990.

[7] P. K. Vitthaladevuni and M.-S. Alouini, "BER computation of 4/M-QAM hierarchical constellations," IEEE Transactions on Broadcasting, vol. 47, no. 3, pp. 228-239, 2001.

[8] W. T. A. Lopes, W. J. L. Queiroz, F. Madeiro, and M. S. Alencar, "Exact bit error probability of M-QAM modulation over flat Rayleigh fading channels," in Proceedings of SBMO/IEEE MTT-S International Microwave and Optoelectronics Conference (IMOC '07), pp. 804-806, Salvador, Brazil, JanuaryNovember 2007.

[9] D. Yoon and K. Cho, "General bit error probability of rectangular quadrature amplitude modulation," Electronics Letters, vol. 38, no. 3, pp. 131-133, 2002.

[10] X. Dong, N. C. Beaulieu, and P. H. Wittke, "Error probabilities of two-dimensional $M$-ary signaling in fading," IEEE Transactions on Communications, vol. 47, no. 3, pp. 352-355, 1999.

[11] J. W. Craig, "A new, simple and exact result for calculating the probability of error for two-dimensional signal constellations," in Proceedings of Military Communications Conference (MILCOM '91), pp. 571-575, November 1991.

[12] A. Maaref and S. Aïssa, "Exact closed-form expression for the bit error rate of orthogonal STBC in Nakagami fading channels," in Proceedings of the 60th IEEE Vehicular Technology 
Conference (VTC'04), pp. 2493-2497, Los Angeles, Calif, USA, September 2004.

[13] A. Maaref, "Performance analysis of orthogonal space-time block codes in spatially correlated MIMO Nakagami fading channels," IEEE Transactions on Wireless Communications, vol. 5, no. 4, pp. 807-817, 2006.

[14] A. Maaref and S. Aïssa, "Adaptive modulation using orthogonal STBC in MIMO Nakagami fading channels," in Proceedings of the IEEE International Symposium on Spread Spectrum Techniques and Applications (ISSSTA '04), pp. 145-149, Sydney, Australia, August-September 2004.

[15] M. G. Shayesteh and A. Aghamohammadi, "On the error probability of linearly modulated signals on frequency-flat Ricean, Rayleigh, and AWGN channels," IEEE Transactions on Communications, vol. 43, no. 2, pp. 1454-1466, 1995.

[16] W. T. A. Lopes, F. Madeiro, and M. S. Alencar, "Closedform expression for the bit error probability of rectangular QAM subject to Rayleigh fading," in Proceedings of the 66th IEEE Vehicular Technology Conference (VTC '07), pp. 915-919, Baltimore, Md, USA, September-October 2007.

[17] W. T. A. Lopes and M. S. Alencar, "QPSK detection schemes for Rayleigh fading channels," in Proceedings of the IEEE International Telecommunications Symposium (ITS '02), Natal, Brazil, September 2002.

[18] J. M. Wozencraft and I. M. Jacobs, Principles of Communication Engineering, John Wiley \& Sons, New York, NY, USA, 1965.

[19] A. Papoulis, Probability, Random Variables and Stochastic Processes, McGraw-Hill, New York, NY, USA, 1991.

[20] W. J. L. Queiroz, W. T. A. Lopes, F. Madeiro, and M. S. Alencar, "Bit error probability of $M$-QAM and $I \times J$-QAM modulation schemes in Nakagami fading," in Proceedings of the SBMO/IEEE MTT-S International Microwave and Optoelectronics Conference (IMOC '07), pp. 807-811, Salvador, Brazil, January-November 2007.

[21] M. Abramowitz and I. A. Stegun, Handbook of Mathematical Functions, Dover, New York, NY, USA, 1972.

[22] T. Q. Duong, H. Shin, and E.-K. Hong, "Error probability of binary and $M$-ary signals with spatial diversity in Nakagamiq (Hoyt) fading channels," EURASIP Journal on Wireless Communications and Networking, vol. 2007, Article ID 53742, 8 pages, 2007.

[23] W. T. A. Lopes, F. Madeiro, and M. S. Alencar, "Um Novo Método para o Cálculo da Probabilidade de Erro de Bit de Esquemas de Modulação QAM Sujeitos ao Desvanecimento Rayleigh," Revista da Sociedade Brasileira de Telecomunicações, vol. 19, no. 2, pp. 58-67, 2004.

[24] D. E. Knuth, The Art of Computer Programming: Seminumerical Algorithms, vol. 2, chapter 4, Addison-Wesley, Reading, Mass, USA, 1969. 